Kragujevac Journal of Mathematics

Volume 44(4) (2020), Pages 617-637.

\title{
EXISTENCE OF RENORMALIZED SOLUTIONS FOR SOME ANISOTROPIC QUASILINEAR ELLIPTIC EQUATIONS
}

\author{
T. AHMEDATT ${ }^{1}$, A. AHMED ${ }^{1}$, H. HJIAJ ${ }^{2}$, AND A. TOUZANI ${ }^{1}$
}

\begin{abstract}
In this paper, we consider a class of anisotropic quasilinear elliptic
\end{abstract} equations of the type

$$
\begin{cases}-\sum_{\substack{i=1 \\ u=0}}^{N} \partial_{i}(x, u, \nabla u)+|u|^{s(x)-1} u=f(x, u), & \text { in } \Omega, \\ \text { on } \partial \Omega,\end{cases}
$$

where $f(x, s)$ is a Carathéodory function which satisfies some growth condition. We prove the existence of renormalized solutions for our Dirichlet problem, and some regularity results are concluded.

\section{INTRODUCTION}

Let $\Omega$ be an open bounded subset of $\mathbb{R}^{N}, N \geq 2$, with the smooth boundary $\partial \Omega$. Zhao et al. have studied in [17] the quasilinear elliptic problem

$$
\begin{cases}-\operatorname{div}(a(x, \nabla u))+|u|^{p-2} u=\lambda f(x, u), & \text { in } \Omega, \\ \int_{\partial \Omega} a(x, \nabla u) \cdot n d s=0, & \text { on } \partial \Omega,\end{cases}
$$

They have proved the existence of weak solutions under some suitable growth assumptions on $f(x, s)$, (see also $[2,7]$ ). In the framework of Sobolev spaces with variable exponents, Fan and Zhang [11] have considered the following nonlinear elliptic problem

$$
\begin{cases}\operatorname{div}\left(|\nabla u|^{p(x)-2} \nabla u\right)=\lambda f(x, u), & \text { in } \Omega, \\ u=0, & \text { on } \partial \Omega,\end{cases}
$$

Key words and phrases. Anisotropic Sobolev spaces, variable exponents, quasilinear elliptic equations, renormalized solutions.

2010 Mathematics Subject Classification. Primary: 35J62. Secondary: 35J20.

DOI 10.46793/KgJMat2004.617A

Received: June 06, 2018.

Accepted: August 21, 2018. 
where $\lambda>0$ and $f(x, s)$ satisfies the growth condition $|f(x, s)| \leq \eta+\theta|s|^{\delta-1}$, where $1 \leq \delta \leq p^{-}$and $\eta, \theta$ are two positive constants (we refer also to [6]). In [3], the authors have proved the existence of weak solutions for the quasilinear $p(x)$-elliptic problem

$$
-\operatorname{div} a(x, u, \nabla u)=f(x, u, \nabla u)
$$

by using the calculus of variations operators method, where $f(x, s, \xi)$ is a Carathéodory function which satisfies some growth condition.

In the framework of anisotropic Sobolev spaces, Di Nardo, Feo and Guibé have studied in [9] the existence of renormalized solutions for some class of nonlinear anisotropic elliptic problems of the type

$$
-\sum_{i=1}^{N} \partial_{x_{i}}\left(a_{i}(x, u)\left|\partial_{x_{i}} u\right|^{p_{i}-2} \partial_{x_{i}} u\right)=f-\operatorname{div} g, \quad \text { in } \Omega,
$$

with $f \in L^{1}(\Omega)$ and $g \in \Pi_{i=1}^{N} L^{p_{i}^{\prime}}(\Omega)$, the uniqueness of renormalized solution was concluded under some local Lipschitz conditions on the function $a_{i}(x, s)$ with respect to $s$, (see also [1] and [8]).

The aim of this paper is to study the existence and regularity of renormalized solutions for the anisotropic quasilinear elliptic problem

$$
\begin{cases}-\sum_{i=1}^{N} \partial^{i} a_{i}(x, u, \nabla u)+|u|^{s(x)-1} u=f(x, u), & \text { in } \Omega \\ u=0, & \text { on } \partial \Omega\end{cases}
$$

where $\left(a_{i}(x, s, \xi)\right)_{i=1, \ldots, N}$ are Carathéodory functions, the right-hand side $f(x, s)$ is a Carathéodory function satisfying only some nonstandard growth condition.

One of our motivations for studying (1.1) comes from these applications to electrorheological fluids as an important class of non-Newtonian fluids (sometimes referred to as smart fluids). The electro-rheological fluids are characterized by their ability to drastically change the mechanical properties under the influence of an external electromagnetic field. A mathematical model of electro-rheological fluids was proposed in $[14,15]$, also in the robotics and space technology (we refer for example to [16]).

One of the difficulties in proving the existence of renormalized solutions stems from the nonstandard growth of the Carathéodory function $f(x, s)$, to overcome the difficulty, we use the regularizing effect of the term $|u|^{s(x)-1} u$ with some special technics.

The rest of this paper is structured as follows. In Section 2 we recall some definitions and results on the anisotropic variable exponent Sobolev spaces. We introduce in Section 3 some assumptions for which our problem has at least one renormalized solution. Section 4 will be devoted to show the existence of renormalized solutions $u$ for the problem (1.1) in the anisotropic Sobolev space with variable exponents, and we will give some regularity results, that is $|u|^{s(x)-1} u \in L^{1}(\Omega)$. 


\section{Preliminary}

Let $\Omega$ be a bounded open subset of $\mathbb{R}^{N}, N \geq 2$, we denote

$$
\mathcal{C}_{+}(\Omega)=\left\{\text { measurable function } p(\cdot): \Omega \rightarrow \mathbb{R} \text { such that } 1<p^{-} \leq p^{+}<N\right\},
$$

where

$$
p^{-}=\operatorname{ess} \inf \{p(x) / x \in \Omega\} \text { and } p^{+}=\operatorname{ess} \sup \{p(x) / x \in \Omega\} .
$$

We define the Lebesgue space with variable exponent $L^{p(\cdot)}(\Omega)$ as the set of all measurable functions $u: \Omega \rightarrow \mathbb{R}$ for which the convex modular

$$
\rho_{p(\cdot)}(u):=\int_{\Omega}|u|^{p(x)} d x
$$

is finite. If the exponent is bounded, i.e., if $p^{+}<+\infty$, then the expression

$$
\|u\|_{p(\cdot)}=\inf \left\{\lambda>0: \rho_{p(\cdot)}(u / \lambda) \leq 1\right\}
$$

defines a norm in $L^{p(\cdot)}(\Omega)$, called the Luxemburg norm. The space $\left(L^{p(\cdot)}(\Omega),\|\cdot\|_{p(\cdot)}\right)$ is a separable Banach space. Moreover, if $1<p^{-} \leq p^{+}<+\infty$, then $L^{p(\cdot)}(\Omega)$ is uniformly convex, hence reflexive, and its dual space is isomorphic to $L^{p^{\prime}(\cdot)}(\Omega)$, where $\frac{1}{p(x)}+\frac{1}{p^{\prime}(x)}=1$. Finally, we have the Hölder type inequality:

$$
\left|\int_{\Omega} u v d x\right| \leq\left(\frac{1}{p^{-}}+\frac{1}{\left(p^{\prime}\right)^{-}}\right)\|u\|_{p(\cdot)}\|v\|_{p^{\prime}(\cdot)},
$$

for any $u \in L^{p(\cdot)}(\Omega)$ and $v \in L^{p^{\prime}(\cdot)}(\Omega)$.

The Sobolev space with variable exponent $W^{1, p(\cdot)}(\Omega)$ is defined by

$$
W^{1, p(\cdot)}(\Omega)=\left\{u \in L^{p(\cdot)}(\Omega) \text { and }|\nabla u| \in L^{p(\cdot)}(\Omega)\right\},
$$

which is a Banach space, equipped with the following norm

$$
\|u\|_{1, p(\cdot)}=\|u\|_{p(\cdot)}+\|\nabla u\|_{p(\cdot)} .
$$

The space $\left(W^{1, p(\cdot)}(\Omega),\|\cdot\|_{1, p(\cdot)}\right)$ is a separable and reflexive Banach space. We define $W_{0}^{1, p(\cdot)}(\Omega)$ as the closure of $\mathcal{C}_{0}^{\infty}(\Omega)$ in $W^{1, p(\cdot)}(\Omega)$. For more details on variable exponent Lebesgue and Sobolev spaces, we refer the reader to [10].

Now, we present the anisotropic variable exponent Sobolev space, used in the study of our quasilinear anisotropic elliptic problem.

Let $p_{1}(\cdot), p_{2}(\cdot), \ldots, p_{N}(\cdot)$ be $N$ variable exponents in $\mathcal{C}_{+}(\Omega)$. We denote

$$
\vec{p}(\cdot)=\left(p_{1}(\cdot), \ldots, p_{N}(\cdot)\right) \text { and } D^{i} u=\frac{\partial u}{\partial x_{i}}, \quad \text { for } i=1, \ldots, N,
$$

and we define

$$
\underline{p}^{+}=\max \left\{p_{1}^{-}, \ldots, p_{N}^{-}\right\} \text {and } \underline{p}^{-}=\min \left\{p_{1}^{-}, \ldots, p_{N}^{-}\right\}, \quad \text { then } 1<\underline{p}^{-} \leq \underline{p}^{+} .
$$

The anisotropic variable exponent Sobolev space $W^{1, \vec{p}(\cdot)}(\Omega)$ is defined as follow

$$
W^{1, \vec{p}(\cdot)}(\Omega)=\left\{u \in W^{1,1}(\Omega) \text { and } D^{i} u \in L^{p_{i}(\cdot)}(\Omega) \text { for } i=1,2, \ldots, N\right\},
$$


endowed with the norm

$$
\|u\|_{1, \vec{p}(\cdot)}=\|u\|_{1,1}+\sum_{i=1}^{N}\left\|D^{i} u\right\|_{p_{i}(\cdot)} .
$$

We define also $W_{0}^{1, \vec{p}(\cdot)}(\Omega)$ as the closure of $\mathcal{C}_{0}^{\infty}(\Omega)$ in $W^{1, \vec{p}(\cdot)}(\Omega)$ with respect to the norm (2.1). The space $\left(W_{0}^{1, \vec{p}(\cdot)}(\Omega),\|u\|_{1, \vec{p}(\cdot)}\right)$ is a reflexive Banach space (cf. [13]).

Remark 2.1. In view of the continuous embedding $W_{0}^{1, \vec{p}(\cdot)}(\Omega) \hookrightarrow W_{0}^{1,1}(\Omega)$ and the Poincaré type inequality we conclude that the two norms $\|u\|_{1, \vec{p}(\cdot)}$ and $\sum_{i=1}^{N}\left\|D^{i} u\right\|_{p_{i}(\cdot)}$ are equivalent in the anisotropic variable exponent Sobolev spaces.

Lemma 2.1. We have the following continuous and compact embeddings.

- If $\underline{p}^{-}<N$, then $W_{0}^{1, \vec{p}(\cdot)}(\Omega) \hookrightarrow \hookrightarrow L^{q}(\Omega)$, for $q \in\left[\underline{p}^{-}, \underline{p}^{*}\left[\right.\right.$, where $\underline{p}^{*}=\frac{N \underline{p}^{-}}{N-\underline{p}^{-}}$.

- If $\underline{p}^{-}=N$, then $W_{0}^{1, \vec{p}(\cdot)}(\Omega) \hookrightarrow \hookrightarrow L^{q}(\Omega)$, for all $q \in\left[\underline{p}^{-},+\infty[\right.$.

- If $\underline{p}^{-}>N$, then $W_{0}^{1, \vec{p}(\cdot)}(\Omega) \hookrightarrow \hookrightarrow L^{\infty}(\Omega) \cap \mathrm{e}^{0}(\bar{\Omega})$.

The proof of this lemma follows from the fact that the embedding $W_{0}^{1, \vec{p}(\cdot)}(\Omega) \hookrightarrow$ $W_{0}^{1, \underline{p}^{-}}(\Omega)$ is continuous, and in view of the compact embedding theorem for Sobolev spaces.

Proposition 2.1. The dual of $W_{0}^{1, \vec{p}(\cdot)}(\Omega)$ is denote by $W^{-1, \vec{p}^{\prime}(\cdot)}(\Omega)$, where $\overrightarrow{p^{\prime}}(\cdot)=$ $\left(p_{1}^{\prime}(\cdot), \ldots, p_{N}^{\prime}(\cdot)\right)$ and $\frac{1}{p_{i}^{\prime}(x)}+\frac{1}{p_{i}(x)}=1$ (cf. [5] for the constant exponent case). For each $F \in W^{-1, \overrightarrow{p^{\prime}}(\cdot)}(\Omega)$ there exists $F_{0} \in\left(L^{\underline{p}^{+}}(\Omega)\right)^{\prime}$ and $F_{i} \in L^{p_{i}^{\prime}(\cdot)}(\Omega)$ for $i=1,2, \ldots, N$, such that $F=F_{0}-\sum_{i=1}^{N} D^{i} F_{i}$. Moreover, for any $u \in W_{0}^{1, \vec{p}(\cdot)}(\Omega)$, we have

$$
\langle F, u\rangle=\sum_{i=0}^{N} \int_{\Omega} F_{i} D^{i} u d x .
$$

We define a norm on the dual space by

$$
\begin{aligned}
\|F\|_{-1, \overrightarrow{p^{\prime}}(\cdot)}=\inf \{ & \sum_{i=0}^{N}\left\|F_{i}\right\|_{p_{i}^{\prime}(\cdot)} \text { with } F=F_{0}-\sum_{i=1}^{N} D^{i} F_{i} \text { such that } F_{0} \in\left(L^{\underline{p}^{+}}(\Omega)\right)^{\prime} \\
& \text { and } \left.F_{i} \in L^{p_{i}^{\prime}(\cdot)}(\Omega)\right\} .
\end{aligned}
$$

Definition 2.1. Let $k>0$, the truncation function $T_{k}(\cdot): \mathbb{R} \rightarrow \mathbb{R}$ is defined by

$$
T_{k}(s)= \begin{cases}s, & \text { if }|s| \leq k, \\ k \frac{s}{|s|}, & \text { if }|s|>k,\end{cases}
$$

and we define

$$
\mathcal{T}_{0}^{1, \vec{p}(\cdot)}(\Omega):=\left\{u: \Omega \rightarrow \mathbb{R} \text { measurable, such that } T_{k}(u) \in W_{0}^{1, \vec{p}(\cdot)}(\Omega) \text { for any } k>0\right\} .
$$


Proposition 2.2. Let $u \in \mathcal{T}_{0}^{1, \vec{p}(\cdot)}(\Omega)$. For any $i \in\{1, \ldots, N\}$, there exists a unique measurable function $v_{i}: \Omega \rightarrow \mathbb{R}$ such that

$$
D^{i} T_{k}(u)=v_{i} \cdot \chi_{\{|u|<k\}} \text { a.e. } x \in \Omega, \text { for all } k>0,
$$

where $\chi_{A}$ denotes the characteristic function of a measurable set $A$. The functions $v_{i}$ are called the weak partial derivatives of $u$ and are still denoted $D^{i} u$. Moreover, if $u$ belongs to $W_{0}^{1,1}(\Omega)$, then $v_{i}$ coincides with the standard distributional derivative of $u$, that is, $v_{i}=D^{i} u$.

\section{Essential Assumptions}

Let $\Omega$ be a bounded open subset of $\mathbb{R}^{N}(N \geq 2)$. We consider $\vec{p}(\cdot)=\left(p_{1}(\cdot), \ldots, p_{N}(\cdot)\right)$ the vector of exponents $p_{i}(\cdot) \in C_{+}(\Omega)$ for $i=1, \ldots, N$, and let $q(\cdot), s(\cdot) \in C_{+}(\Omega)$ where

$$
q(x)<\max \left(s(x), \underline{p}^{+}-1\right) \text { a.e. in } \Omega .
$$

We consider the Leray-Lions operator $A$ acted from $W_{0}^{1, \vec{p}(\cdot)}(\Omega)$ into its dual $W^{-1, \vec{p}^{\prime}(\cdot)}(\Omega)$, defined by the formula

$$
A u=-\sum_{i=1}^{N} \partial^{i} a_{i}(x, u, \nabla u),
$$

where $a_{i}: \Omega \times \mathbb{R} \times \mathbb{R}^{N} \rightarrow \mathbb{R}$ are Carathéodory function which satisfy the following conditions

$$
\begin{aligned}
& \left|a_{i}(x, s, \xi)\right| \leq \beta\left(K_{i}(x)+|s|^{p_{i}(x)-1}+|\xi|^{p_{i}(x)-1}\right), \quad \text { for any } i=1, \ldots, N, \\
& a_{i}(x, s, \xi) \xi_{i} \geq \alpha\left|\xi_{i}\right|^{p_{i}(x)}, \quad \text { for any } i=1, \ldots, N
\end{aligned}
$$

for all $\xi=\left(\xi_{1}, \ldots, \xi_{N}\right)$ and $\xi^{\prime}=\left(\xi_{1}^{\prime}, \ldots, \xi_{N}^{\prime}\right)$, we have

$$
\left[a_{i}(x, s, \xi)-a_{i}\left(x, s, \xi^{\prime}\right)\right]\left(\xi_{i}-\xi_{i}^{\prime}\right)>0, \quad \text { for } \xi_{i} \neq \xi_{i}^{\prime}
$$

for a.e. $x \in \Omega$, all $(s, \xi) \in \mathbb{R} \times \mathbb{R}^{N}$, where $K_{i}(x)$ is a positive function lying in $L^{p_{i}^{\prime}(\cdot)}(\Omega)$ and $\alpha, \beta>0$.

As a consequence of $(3.2)$ and the continuity of the function $a_{i}(x, s, \cdot)$ with respect to $\xi$, we have

$$
a_{i}(x, s, 0)=0 \text {. }
$$

In this paper, we consider the following quasilinear anisotropic elliptic problem

$$
\begin{cases}-\sum_{i=1}^{N} \partial^{i} a_{i}(x, u, \nabla u)+|u|^{s(x)-1} u=f(x, u), & \text { in } \Omega \\ u=0, & \text { on } \partial \Omega\end{cases}
$$

where $f: \Omega \times \mathbb{R} \rightarrow \mathbb{R}$ is a Carathéodory function satisfying

$$
|f(x, r)| \leq g(x)+|r|^{q(x)} \text { a.e in } \Omega,
$$

and $g(\cdot)$ is a measurable positive function in $L^{1}(\Omega)$. 
Remark 3.1. The assumption (3.1) is used here to ensure that $a_{i}(x, u, \nabla u)$ belongs to $L^{p_{i}^{\prime}(\cdot)}(\Omega)$. In the other case where $A u=-\sum_{i=1}^{N} \partial^{i} a_{i}(x, \nabla u)$, the uniqueness of solution can be concluded under some additional conditions on the Carathéodory function $f(x, s)$.

\section{Main Results}

We begin by recalling some important lemmas useful to prove our main result.

Lemma $4.1([3])$. Let $g \in L^{r(\cdot)}(\Omega)$ and $g_{n} \in L^{r(\cdot)}(\Omega)$ with $\left\|g_{n}\right\|_{r(\cdot)} \leq C$ for $1<r(x)<$ $\infty$. If $g_{n}(x) \rightarrow g(x)$ a.e. on $\Omega$, then $g_{n} \rightarrow g$ in $L^{r(\cdot)}(\Omega)$.

Lemma 4.2 ([4]). Assuming that (3.1)-(3.3) hold, and let $\left(u_{n}\right)_{n \in \mathbb{N}}$ be a sequence in $W_{0}^{1, \vec{p}(\cdot)}(\Omega)$ such that $u_{n} \rightarrow u$ in $W_{0}^{1, \vec{p}(\cdot)}(\Omega)$ and

$$
\begin{aligned}
& \int_{\Omega}\left(\left|u_{n}\right|^{p_{0}(x)-2} u_{n}-|u|^{p_{0}(x)-2} u\right)\left(u_{n}-u\right) d x \\
& +\sum_{i=1}^{N} \int_{\Omega}\left(a_{i}\left(x, u_{n}, \nabla u_{n}\right)-a_{i}\left(x, u_{n}, \nabla u\right)\right)\left(D^{i} u_{n}-D^{i} u\right) d x \rightarrow 0,
\end{aligned}
$$

then $u_{n} \rightarrow u$ in $W_{0}^{1, \vec{p}(\cdot)}(\Omega)$ for a subsequence.

Our objective is to prove the existence of renormalized solutions for the quasilinear anisotropic elliptic problem (3.4).

Definition 4.1. A measurable function $u$ is called renormalized solution of the quasilinear elliptic problem $(3.4)$ if $T_{k}(u) \in W_{0}^{1, \vec{p}(\cdot)}(\Omega)$ for any $k>0$, with $f(x, u) \in L^{1}(\Omega)$, and

$$
\lim _{h \rightarrow \infty} \sum_{i=1}^{N} \int_{\{h<|u| \leq h+1\}} a_{i}(x, u, \nabla u) D^{i} u d x=0,
$$

such that $u$ satisfies the following equality

$$
\begin{aligned}
& \sum_{i=1}^{N} \int_{\Omega} a_{i}(x, u, \nabla u)\left(S^{\prime}(u) \varphi D^{i} u+S(u) D^{i} \varphi\right) d x+\int_{\Omega}|u|^{s(x)-1} u S(u) \varphi d x \\
= & \int_{\Omega} f(x, u) S(u) \varphi d x,
\end{aligned}
$$

for every $\varphi \in W_{0}^{1, \vec{p}(\cdot)}(\Omega) \cap L^{\infty}(\Omega)$ and for any smooth function $S(\cdot) \in W^{1, \infty}(\mathbb{R})$ with a compact support.

Theorem 4.1. Assuming that the conditions (3.1)-(3.3) and (3.5) hold true, then the quasilinear anisotropic elliptic problem (3.4) has at least one renormalized solution. Moreover, we have

$$
|u|^{s(x)} \in L^{1}(\Omega) .
$$




\subsection{Proof of Theorem 4.1.}

Step 1: approximate problems. Firstly, we consider the approximate problem

$$
\begin{cases}A_{n} u_{n}+\left|T_{n}\left(u_{n}\right)\right|^{s(x)-1} T_{n}\left(u_{n}\right)=f_{n}\left(x, T_{n}\left(u_{n}\right)\right), & \text { in } \Omega, \\ u_{n}=0, & \text { on } \partial \Omega,\end{cases}
$$

where $A_{n} v=-\sum_{i=1}^{N} \partial^{i} a_{i}\left(x, T_{n}(v), \nabla v\right)$ and $f_{n}(x, r)=T_{n}(f(x, r))$. Thanks to (3.5), it's clear that

$$
\left|f_{n}(x, r)\right| \leq n \text { and }\left|f_{n}(x, r)\right| \leq g(x)+|r|^{q(x)} .
$$

We consider the operator $G_{n}: W_{0}^{1, \vec{p}(\cdot)}(\Omega) \rightarrow W^{-1, \vec{p}^{\prime} \cdot \cdot(\Omega)}$ by

$$
\left\langle G_{n} u, v\right\rangle=\int_{\Omega}\left|T_{n}(u)\right|^{s(x)-1} T_{n}(u) v d x-\int_{\Omega} f_{n}\left(x, T_{n}(u)\right) v d x,
$$

for any $u, v \in W_{0}^{1, \vec{p}(\cdot)}(\Omega)$. In view of the generalized Hölder-type inequality, we have

$$
\begin{aligned}
\left|\left\langle G_{n} u, v\right\rangle\right| & \leq \int_{\Omega}\left|T_{n}(u)\right|^{s(x)}|v| d x+\int_{\Omega}\left|f_{n}\left(x, T_{n}(u)\right)\right||v| d x \\
& \leq n^{s^{+}} \int_{\Omega}|v| d x+n \int_{\Omega}|v| d x \\
& =\left(n^{s^{+}}+n\right)\|v\|_{1} \\
& \leq C_{1}\|v\|_{1, \vec{p}(\cdot) .}
\end{aligned}
$$

Lemma 4.3. The bounded operator $B_{n}=A_{n}+G_{n}$ acted from $W_{0}^{1, \vec{p}(\cdot)}(\Omega)$ into $W^{-1, p^{\prime}(\cdot)}(\Omega)$ is pseudo-monotone. Moreover, $B_{n}$ is coercive in the following sense:

$$
\frac{\left\langle B_{n} v, v\right\rangle}{\|v\|_{1, \vec{p}(\cdot)}} \rightarrow+\infty \text { as }\|v\|_{1, \vec{p}(\cdot)} \rightarrow \infty, \quad \text { for any } v \in W_{0}^{1, \vec{p}(\cdot)}(\Omega) .
$$

Proof. In view of the Hölder's inequality and the growth condition (3.1), it's easy to see that the operator $A_{n}$ is bounded, and by (4.3) we conclude that $B_{n}$ is bounded. For the coercivity, we have for any $u \in W_{0}^{1, \vec{p}(\cdot)}(\Omega)$,

$$
\begin{aligned}
\left\langle B_{n} u, u\right\rangle= & \left\langle A_{n} u, u\right\rangle+\left\langle G_{n} u, u\right\rangle \\
= & \sum_{i=1}^{N} \int_{\Omega} a_{i}\left(x, T_{n}(u), \nabla u\right) D^{i} u d x+\int_{\Omega}\left|T_{n}(u)\right|^{s(x)}|u| d x \\
& -\int_{\Omega}\left|f_{n}\left(x, T_{n}(u)\right) \| u\right| d x \\
\geq & \alpha \sum_{i=1}^{N} \int_{\Omega}\left|D^{i} u\right|^{p_{i}(x)} d x+\int_{\Omega}\left|T_{n}(u)\right|^{s(x)+1} d x-C_{2} n\|u\|_{p_{0}(\cdot)} \\
\geq & C_{0}\|u\|_{1, \vec{p}(\cdot)}^{\frac{p^{-}}{}}-\alpha N|\Omega|-C_{2} n\|u\|_{1, \vec{p}(\cdot),}
\end{aligned}
$$

it follows that

$$
\frac{\left\langle B_{n} u, u\right\rangle}{\|u\|_{1, \vec{p}(\cdot)}} \rightarrow+\infty \text { as }\|u\|_{1, \vec{p}(\cdot)} \rightarrow \infty
$$


It remains to show that $B_{n}$ is pseudo-monotone. Let $\left(u_{k}\right)_{k \in \mathbb{N}}$ be a sequence in $W_{0}^{1, \vec{p}(\cdot)}(\Omega)$ such that

$$
\left\{\begin{array}{l}
u_{k} \rightarrow u, \quad \text { in } W_{0}^{1, \vec{p}(\cdot)}(\Omega), \\
B_{n} u_{k} \rightarrow \chi_{n}, \quad \text { in } W^{-1, \vec{p}^{\prime}(\cdot)}(\Omega), \\
\limsup _{k \rightarrow \infty}\left\langle B_{n} u_{k}, u_{k}\right\rangle \leq\left\langle\chi_{n}, u\right\rangle
\end{array}\right.
$$

We will prove that

$$
\chi_{n}=B_{n} u \text { and }\left\langle B_{n} u_{k}, u_{k}\right\rangle \rightarrow\left\langle\chi_{n}, u\right\rangle \text { as } k \rightarrow \infty .
$$

In view of the compact embedding $W_{0}^{1, \vec{p}(\cdot)}(\Omega) \hookrightarrow \hookrightarrow L^{1}(\Omega)$, we have $u_{k} \rightarrow u$ in $L^{1}(\Omega)$ and a.e. $\Omega$, for a subsequence still denoted $\left(u_{k}\right)_{k \in \mathbb{N}}$.

We have $\left(u_{k}\right)_{k \in \mathbb{N}}$ is a bounded sequence in $W_{0}^{1, \vec{p}(\cdot)}(\Omega)$, using the growth condition (3.1) it's clear that the sequence $\left(a_{i}\left(x, T_{n}\left(u_{k}\right), \nabla u_{k}\right)\right)_{k \in \mathbb{N}}$ is bounded in $L^{p_{i}^{\prime}(\cdot)}(\Omega)$, then there exists a function $\varphi_{i} \in L^{p_{i}^{\prime}(\cdot)}(\Omega)$ such that

$$
a_{i}\left(x, T_{n}\left(u_{k}\right), \nabla u_{k}\right) \rightarrow \varphi_{i} \text { in } L^{p_{i}^{\prime}(\cdot)}(\Omega) \text { as } k \rightarrow \infty .
$$

On the one hand we have

$$
\left|T_{n}\left(u_{k}\right)\right|^{s(x)-1} T_{n}\left(u_{k}\right) \rightarrow\left|T_{n}(u)\right|^{s(x)-1} T_{n}(u) \text { weak-* in } L^{\infty}(\Omega),
$$

and since $f_{n}\left(x, T_{n}(s)\right)$ is a Carathéodory function, then

$$
f_{n}\left(x, T_{n}\left(u_{k}\right)\right) \rightarrow f_{n}\left(x, T_{n}(u)\right) \text { weak-* in } L^{\infty}(\Omega) .
$$

Then, for any $v \in W_{0}^{1, \vec{p}(\cdot)}(\Omega)$ we have

$$
\begin{aligned}
\left\langle\chi_{n}, v\right\rangle= & \lim _{k \rightarrow \infty}\left\langle B_{n} u_{k}, v\right\rangle \\
= & \lim _{k \rightarrow \infty} \sum_{i=1}^{N} \int_{\Omega} a_{i}\left(x, T_{n}\left(u_{k}\right), \nabla u_{k}\right) D^{i} v d x+\lim _{k \rightarrow \infty} \int_{\Omega}\left|T_{n}\left(u_{k}\right)\right|^{s(x)-1} T_{n}\left(u_{k}\right) v d x \\
& -\lim _{k \rightarrow \infty} \int_{\Omega} f_{n}\left(x, T_{n}\left(u_{k}\right)\right) v d x \\
= & \sum_{i=1}^{N} \int_{\Omega} \varphi_{i} D^{i} v d x+\int_{\Omega}\left|T_{n}(u)\right|^{s(x)-1} T_{n}(u) v d x-\int_{\Omega} f_{n}\left(x, T_{n}(u)\right) v d x .
\end{aligned}
$$

Having in mind (4.4) and (4.8), we conclude that

$$
\begin{aligned}
\limsup _{k \rightarrow \infty}\left\langle B_{n}\left(u_{k}\right), u_{k}\right\rangle= & \limsup _{k \rightarrow \infty}\left(\sum_{i=1}^{N} \int_{\Omega} a_{i}\left(x, T_{n}\left(u_{k}\right), \nabla u_{k}\right) D^{i} u_{k} d x\right. \\
& \left.+\int_{\Omega}\left|T_{n}\left(u_{k}\right)\right|^{s(x)-1} T_{n}\left(u_{k}\right) u_{k} d x-\int_{\Omega} f_{n}\left(x, T_{n}\left(u_{k}\right)\right) u_{k} d x\right) \\
\leq & \sum_{i=1}^{N} \int_{\Omega} \varphi_{i} D^{i} u d x+\int_{\Omega}\left|T_{n}(u)\right|^{s(x)-1} T_{n}(u) u d x \\
& -\int_{\Omega} f_{n}\left(x, T_{n}(u)\right) u d x .
\end{aligned}
$$


Since $u_{k} \rightarrow u$ strongly in $L^{1}(\Omega)$, and thanks to (4.6)-(4.7) we obtain

$$
\int_{\Omega}\left|T_{n}\left(u_{k}\right)\right|^{s(x)-1} T_{n}\left(u_{k}\right) u_{k} d x \rightarrow \int_{\Omega}\left|T_{n}(u)\right|^{s(x)-1} T_{n}(u) u d x
$$

and

$$
\int_{\Omega} f_{n}\left(x, T_{n}\left(u_{k}\right)\right) u_{k} d x \rightarrow \int_{\Omega} f_{n}\left(x, T_{n}(u)\right) u d x .
$$

Therefore,

$$
\limsup _{k \rightarrow \infty} \sum_{i=1}^{N} \int_{\Omega} a_{i}\left(x, T_{n}\left(u_{k}\right), \nabla u_{k}\right) D^{i} u_{k} d x \leq \sum_{i=1}^{N} \int_{\Omega} \varphi_{i} D^{i} u d x .
$$

On the other hand, in view of (3.3) we have

$$
\sum_{i=1}^{N} \int_{\Omega}\left(a_{i}\left(x, T_{n}\left(u_{k}\right), \nabla u_{k}\right)-a_{i}\left(x, T_{n}\left(u_{k}\right), \nabla u\right)\right)\left(D^{i} u_{k}-D^{i} u\right) d x \geq 0,
$$

then

$$
\begin{aligned}
\sum_{i=1}^{N} \int_{\Omega} a_{i}\left(x, T_{n}\left(u_{k}\right), \nabla u_{k}\right) D^{i} u_{k} d x \geq & \sum_{i=1}^{N} \int_{\Omega} a_{i}\left(x, T_{n}\left(u_{k}\right), \nabla u_{k}\right) D^{i} u d x \\
& +\sum_{i=1}^{N} \int_{\Omega} a_{i}\left(x, T_{n}\left(u_{k}\right), \nabla u\right)\left(D^{i} u_{k}-D^{i} u\right) d x .
\end{aligned}
$$

In view of Lebesgue's dominated convergence theorem we have $T_{n}\left(u_{k}\right) \rightarrow T_{n}(u)$ in $L^{p_{i}(\cdot)}(\Omega)$, thus $a_{i}\left(x, T_{n}\left(u_{k}\right), \nabla u\right) \rightarrow a_{i}\left(x, T_{n}(u), \nabla u\right)$ strongly in $L^{p_{i}^{\prime}(\cdot)}(\Omega)$, and using (4.5) we get

$$
\liminf _{k \rightarrow \infty} \sum_{i=1}^{N} \int_{\Omega} a_{i}\left(x, T_{n}\left(u_{k}\right), \nabla u_{k}\right) D^{i} u_{k} d x \geq \sum_{i=1}^{N} \int_{\Omega} \varphi_{i} D^{i} u d x .
$$

Having in mind (4.11), we conclude that

$$
\lim _{k \rightarrow \infty} \sum_{i=1}^{N} \int_{\Omega} a_{i}\left(x, T_{n}\left(u_{k}\right), \nabla u_{k}\right) D^{i} u_{k} d x=\sum_{i=1}^{N} \int_{\Omega} \varphi_{i} D^{i} u d x .
$$

Therefore, by combining (4.8) and (4.9)-(4.10), we conclude that

$$
\left\langle B_{n} u_{k}, u_{k}\right\rangle \rightarrow\left\langle\chi_{n}, u\right\rangle \text { as } k \rightarrow \infty \text {. }
$$

Now, by (4.13) we can prove that

$$
\begin{aligned}
& \lim _{k \rightarrow \infty}\left(\sum_{i=1}^{N} \int_{\Omega}\left(a_{i}\left(x, T_{n}\left(u_{k}\right), \nabla u_{k}\right)-a_{i}\left(x, T_{n}\left(u_{k}\right), \nabla u\right)\right)\left(D^{i} u_{k}-D^{i} u\right) d x\right. \\
& \left.+\int_{\Omega}\left(\left|u_{k}\right|^{\underline{p}^{+}-2} u_{k}-|u|^{p^{+}-2} u\right)\left(u_{k}-u\right) d x\right)=0,
\end{aligned}
$$

and so, by virtue of Lemma 4.2, we get

$$
u_{k} \rightarrow u \text { in } W_{0}^{1, \vec{p}(\cdot)}(\Omega) \text { and } D^{i} u_{k} \rightarrow D^{i} u \text { a.e. in } \Omega,
$$


then

$$
a_{i}\left(x, T_{n}\left(u_{k}\right), \nabla u_{k}\right) \rightarrow a_{i}\left(x, T_{n}(u), \nabla u\right) \text { in } L^{p_{i}^{\prime}(\cdot)}(\Omega), \quad \text { for } i=1, \ldots, N,
$$

and thanks to (4.6)-(4.7), we obtain $\chi_{n}=B_{n} u$, which conclude the proof of Lemma 4.3.

In view of Lemma 4.3 , there exists at least one weak solution $u_{n} \in W_{0}^{1, \vec{p}(\cdot)}(\Omega)$ of the approximate problem (4.2) (cf. [12], Theorem 2.7, page 180).

Step 2: a priori estimates. Choose $1<\theta<\underline{p}^{-}$such that $1 \leq q(x)<\max \left(s(x), \underline{p}^{+}-\theta\right)$. By taking $\varphi\left(u_{n}\right)=\left(1-\frac{1}{\left(1+\left|u_{n}\right|\right)^{\theta-1}}\right) \operatorname{sign}\left(u_{n}\right) \in W_{0}^{1, \vec{p}(\cdot)}(\Omega)$ as a test function in (4.2), we obtain

$$
\begin{aligned}
& (\theta-1) \sum_{i=1}^{N} \int_{\Omega} \frac{a_{i}\left(x, T_{n}\left(u_{n}\right), \nabla u_{n}\right) \cdot D^{i} u_{n}}{\left(1+\left|u_{n}\right|\right)^{\theta}} d x+\int_{\Omega}\left|T_{n}\left(u_{n}\right)\right|^{s(x)}\left(1-\frac{1}{\left(1+\left|u_{n}\right|\right)^{\theta-1}}\right) d x \\
= & \int_{\Omega} f_{n}\left(x, T_{n}\left(u_{n}\right)\right)\left(1-\frac{1}{\left(1+\left|u_{n}\right|\right)^{\theta-1}}\right) \operatorname{sign}\left(u_{n}\right) d x .
\end{aligned}
$$

By using the coercivity (3.2) and the growth condition (3.5), we obtain

$$
\begin{aligned}
& \alpha(\theta-1) \sum_{i=1}^{N} \int_{\Omega} \frac{\left|D^{i} u_{n}\right|^{p_{i}(x)}}{\left(1+\left|u_{n}\right|\right)^{\theta}} d x+\int_{\Omega}\left|T_{n}\left(u_{n}\right)\right|^{s(x)}\left(1-\frac{1}{\left(1+\left|u_{n}\right|\right)^{\theta-1}}\right) d x \\
\leq & \int_{\Omega}\left(|g(x)|+\left|T_{n}\left(u_{n}\right)\right|^{q(x)}\right)\left(1-\frac{1}{\left(1+\left|u_{n}\right|\right)^{\theta-1}}\right) d x .
\end{aligned}
$$

For the first term on the left hand side of (4.14), for any $i=1, \ldots, N$, we have

$$
\begin{aligned}
\int_{\Omega} \frac{\left|D^{i} u_{n}\right|^{p_{i}(x)}}{\left(1+\left|u_{n}\right|\right)^{\theta}} d x & \geq \int_{\Omega} \frac{\left|D^{i} u_{n}\right|^{p_{i}^{-}}}{\left(1+\left|u_{n}\right|\right)^{\theta}} d x-|\Omega| \\
& =\int_{\Omega}\left|\frac{D^{i} u_{n}}{\left(1+\left|u_{n}\right|\right)^{\frac{\theta}{p_{i}^{-}}}}\right|^{p_{i}^{-}} d x-|\Omega| \\
& =\int_{\Omega}\left|D^{i} \int_{0}^{\left|u_{n}\right|} \frac{d s}{(1+s)^{\frac{\theta}{p_{i}^{-}}}}\right|^{p_{i}^{-}} d x-|\Omega| \\
& \geq \frac{1}{C_{p}} \int_{\Omega}\left|\int_{0}^{\left|u_{n}\right|} \frac{d s}{(1+s)^{\frac{\theta}{p_{i}^{-}}}}\right|^{p_{i}^{-}} d x-|\Omega| \\
& \geq \frac{1}{C_{p}} \int_{\Omega} \frac{\left|u_{n}\right|^{p_{i}^{-}}}{\left(1+\left|u_{n}\right|\right)^{\theta}} d x-|\Omega| \\
& \geq \frac{1}{2^{\theta} C_{p}} \int_{\Omega}^{\left|u_{n}\right|^{p_{i}^{-}}-\theta} d x-2|\Omega|,
\end{aligned}
$$


and since $\varphi\left(u_{n}\right) \geq \frac{1}{2}$ for $\left|u_{n}\right| \geq R$, with $R=2^{\frac{1}{1-\theta}}-1$. Using Young's inequality it follows that

$$
\begin{aligned}
& \frac{\alpha(\theta-1)}{2^{\theta} C_{p}} \sum_{i=1}^{N} \int_{\Omega}\left|u_{n}\right|^{p_{i}^{-}-\theta} d x+\frac{1}{2} \int_{\left\{\left|u_{n}\right| \geq R\right\}}\left|T_{n}\left(u_{n}\right)\right|^{s(x)} d x \\
\leq & \int_{\Omega}|g(x)| d x+\int_{\Omega}\left|T_{n}\left(u_{n}\right)\right|^{q(x)} d x+2 \alpha N(\theta-1)|\Omega| .
\end{aligned}
$$

Since $1 \leq q(x)<\max \left(s(x), p^{+}-\theta\right)$, by using Young's inequality we conclude that (4.16)

$$
\int_{\Omega}\left|T_{n}\left(u_{n}\right)\right|^{q(x)} d x \leq \frac{\alpha(\theta-1)}{2^{\theta+1} C_{p}} \sum_{i=1}^{N} \int_{\Omega}\left|u_{n}\right|^{p_{i}^{-}-\theta} d x+\frac{1}{4} \int_{\left\{\left|u_{n}\right| \geq R\right\}}\left|T_{n}\left(u_{n}\right)\right|^{s(x)} d x+C_{0} .
$$

It follows from (4.15) that there exists a constant $C_{1}$ that does not depend on $n$, such that

$$
\sum_{i=1}^{N} \int_{\Omega}\left|u_{n}\right|^{p_{i}^{-}-\theta} d x+\int_{\Omega}\left|T_{n}\left(u_{n}\right)\right|^{s(x)} d x+\int_{\Omega}\left|T_{n}\left(u_{n}\right)\right|^{q(x)} d x \leq C_{1} .
$$

Let $k \geq 1$, in view of (4.14) we conclude that

$$
\frac{1}{(1+k)^{\theta}} \sum_{i=1}^{N} \int_{\Omega}\left|D^{i} T_{k}\left(u_{n}\right)\right|^{p_{i}(x)} d x \leq \sum_{i=1}^{N} \int_{\Omega} \frac{\left|D^{i} u_{n}\right|^{p_{i}(x)}}{\left(1+\left|u_{n}\right|\right)^{\theta}} d x+\int_{\Omega}\left|T_{n}\left(u_{n}\right)\right|^{s(x)} \leq C_{2} .
$$

Therefore, we obtain

$$
\sum_{i=1}^{N} \int_{\Omega}\left|D^{i} T_{k}\left(u_{n}\right)\right|^{p_{i}(x)} d x \leq C_{2}(1+k)^{\theta}, \quad \text { for } k \geq 1 .
$$

Thus, the sequence $\left(T_{k}\left(u_{n}\right)\right)_{n}$ is bounded in $W_{0}^{1, \vec{p}(\cdot)}(\Omega)$, and there exists a subsequence still denoted $\left(T_{k}\left(u_{n}\right)\right)_{n}$ and $\eta_{k} \in W_{0}^{1, \vec{p}(\cdot)}(\Omega)$ such that

$$
\left\{\begin{array}{l}
T_{k}\left(u_{n}\right) \rightarrow \eta_{k} \text { in } W_{0}^{1, \vec{p}(\cdot)}(\Omega), \\
T_{k}\left(u_{n}\right) \rightarrow \eta_{k} \text { in } L^{1}(\Omega) \text { and a.e. in } \Omega .
\end{array}\right.
$$

On the other hand, in view of Poincaré type inequality, for any $i \in\{1, \ldots, N\}$ we have

$$
\begin{aligned}
k^{p_{i}^{-}} \text {meas }\left\{\left|u_{n}\right|>k\right\} & =\int_{\left\{\left|u_{n}\right|>k\right\}}\left|T_{k}\left(u_{n}\right)\right|^{p_{i}^{-}} d x \leq \int_{\Omega}\left|T_{k}\left(u_{n}\right)\right|^{p_{i}^{-}} d x \\
& \leq C_{p}^{p_{i}^{-}} \int_{\Omega}\left|D^{i} T_{k}\left(u_{n}\right)\right|^{p_{i}^{-}} d x \\
& \leq C_{p}^{p_{i}^{-}} \int_{\Omega}\left|D^{i} T_{k}\left(u_{n}\right)\right|^{p_{i}(x)} d x+C_{p}^{p_{i}^{-}}|\Omega| \\
& \leq \max _{1 \leq i \leq N}\left(C_{p}^{p_{i}^{-}}\right)\left(\sum_{i=1}^{N} \int_{\Omega}\left|D^{i} T_{k}\left(u_{n}\right)\right|^{p_{i}(x)} d x+|\Omega|\right) \\
& \leq C_{3}(1+k)^{\theta},
\end{aligned}
$$


where $C_{3}$ is a constant that does not depend on $k$ and $n$. Since $1<\theta<\underline{p}^{-}$, we conclude that

$$
\operatorname{meas}\left\{\left|u_{n}\right|>k\right\} \leq \frac{C_{3}(1+k)^{\theta}}{k^{p^{+}}} \rightarrow 0 \text { as } k \rightarrow \infty .
$$

Now, we will show that $\left(u_{n}\right)_{n}$ is a Cauchy sequence in measure. Indeed, we have for every $\delta>0$,

$$
\begin{aligned}
\operatorname{meas}\left\{\left|u_{n}-u_{m}\right|>\delta\right\} \leq & \operatorname{meas}\left\{\left|u_{n}\right|>k\right\}+\operatorname{meas}\left\{\left|u_{m}\right|>k\right\} \\
& +\operatorname{meas}\left\{\left|T_{k}\left(u_{n}\right)-T_{k}\left(u_{m}\right)\right|>\delta\right\}
\end{aligned}
$$

Let $\varepsilon>0$, in view of (4.20) we may choose $k=k(\varepsilon)$ large enough such that

$$
\operatorname{meas}\left\{\left|u_{n}\right|>k\right\} \leq \frac{\varepsilon}{3} \text { and } \operatorname{meas}\left\{\left|u_{m}\right|>k\right\} \leq \frac{\varepsilon}{3} .
$$

Moreover, thanks to (4.19) we have

$$
T_{k}\left(u_{n}\right) \rightarrow \eta_{k} \text { in } L^{1}(\Omega) \text { and a.e. in } \Omega \text {. }
$$

Thus $\left(T_{k}\left(u_{n}\right)\right)_{n \in \mathbb{N}}$ is a Cauchy sequence in measure, and for any $k>0$ and $\delta, \varepsilon>0$, there exists $n_{0}=n_{0}(k, \delta, \varepsilon)$ such that

$$
\text { meas }\left\{\left|T_{k}\left(u_{n}\right)-T_{k}\left(u_{m}\right)\right|>\delta\right\} \leq \frac{\varepsilon}{3}, \quad \text { for all } m, n \geq n_{0}(k, \delta, \varepsilon) .
$$

By combining (4.21) and (4.22), we conclude that for all $\delta, \varepsilon>0$, there exists $n_{0}=$ $n_{0}(\delta, \varepsilon)$ such that

$$
\text { meas }\left\{\left|u_{n}-u_{m}\right|>\delta\right\} \leq \varepsilon, \quad \text { for any } n, m \geq n_{0} .
$$

Thus $\left(u_{n}\right)_{n}$ is a Cauchy sequence in measure, and converges almost everywhere, for a subsequence, to some measurable function $u$. Thanks to (4.19) we conclude that

$$
T_{k}\left(u_{n}\right) \rightarrow T_{k}(u) \text { in } W_{0}^{1, \vec{p}(\cdot)}(\Omega) .
$$

In view of Lebesgue dominated convergence theorem, we obtain

$$
T_{k}\left(u_{n}\right) \rightarrow T_{k}(u) \text { in } L^{p_{i}(\cdot)}(\Omega), \quad \text { for } i=1, \ldots, N .
$$

Moreover, by taking $T_{k}\left(u_{n}\right)$ as a test function in the approximate problem (4.2), we have

$$
\begin{aligned}
& \sum_{i=1}^{N} \int_{\Omega} a_{i}\left(x, T_{n}\left(u_{n}\right), \nabla u_{n}\right) D^{i} T_{k}\left(u_{n}\right) d x+\int_{\Omega}\left|T_{n}\left(u_{n}\right)\right|^{s(x)}\left|T_{k}\left(u_{n}\right)\right| d x \\
= & \int_{\Omega} f_{n}\left(x, T_{n}\left(u_{n}\right)\right) T_{k}\left(u_{n}\right) d x .
\end{aligned}
$$

In view of (3.2), (3.5), and using (4.17) we obtain

$$
\begin{aligned}
\alpha \sum_{i=1}^{N} \int_{\Omega}\left|D^{i} T_{k}\left(u_{n}\right)\right|^{p_{i}(x)} d x & \leq \int_{\Omega} g(x)\left|T_{k}\left(u_{n}\right)\right| d x+\int_{\Omega}\left|T_{n}\left(u_{n}\right)\right|^{q(x)}\left|T_{k}\left(u_{n}\right)\right| d x \\
& \leq k\|g(x)\|_{L^{1}(\Omega)}+k\left\|\left|T_{n}\left(u_{n}\right)\right|^{q(x)}\right\|_{L^{1}(\Omega)} \\
& \leq k\left(\|g(x)\|_{L^{1}(\Omega)}+C_{1}\right) .
\end{aligned}
$$


It follows, for any $i=1, \ldots, N$, that

$$
\begin{aligned}
k^{p_{i}^{-}} \operatorname{meas}\left\{\left|u_{n}\right|>k\right\} & \leq \int_{\Omega}\left|T_{k}\left(u_{n}\right)\right|^{p_{i}^{-}} d x \\
& \leq C_{p}^{p_{i}^{-}} \int_{\Omega}\left|D^{i} T_{k}\left(u_{n}\right)\right|^{p_{i}^{-}} d x \\
& \leq C_{p}^{p_{i}^{-}} \int_{\Omega}\left|D^{i} T_{k}\left(u_{n}\right)\right|^{p_{i}(x)} d x+C_{p}^{p_{i}^{-}}|\Omega| \\
& \leq C_{4} k .
\end{aligned}
$$

Thus, we conclude that

$$
k \underline{-}^{p^{+}-1} \cdot \operatorname{meas}\left\{\left|u_{n}\right|>k\right\} \leq C_{4}, \quad \text { for any } k \geq 1,
$$

where $C_{4}$ is a constant that doesn't depend on $k$ and $n$.

Step 3: the equi-integrability of $\left(\left|T_{n}\left(u_{n}\right)\right|^{s(x)-1} T_{n}\left(u_{n}\right)\right)_{n}$ and $\left(f_{n}\left(x, T_{n}\left(u_{n}\right)\right)\right)_{n}$. In the sequel, we denote by $\varepsilon_{i}(n), i=1,2, \ldots$, various real-valued functions of real variables that converge to 0 as $n$ tends to infinity. Similarly, we define $\varepsilon_{i}(h)$ and $\varepsilon_{i}(n, h)$.

In order to pass to the limit in the approximate equation, we shall show that

$$
\left|T_{n}\left(u_{n}\right)\right|^{s(x)-1} T_{n}\left(u_{n}\right) \rightarrow|u|^{s(x)-1} u \text { strongly in } L^{1}(\Omega)
$$

and

$$
f_{n}\left(x, T_{n}\left(u_{n}\right)\right) \rightarrow f(x, u) \text { strongly in } L^{1}(\Omega)
$$

We have $\left|T_{n}\left(u_{n}\right)\right|^{s(x)-1} T_{n}\left(u_{n}\right) \rightarrow|u|^{s(x)-1} u$ and $f_{n}\left(x, T_{n}\left(u_{n}\right)\right) \rightarrow f(x, u)$ a.e. in $\Omega$. Thus, in view of Vitali's theorem, to show the convergence $(4.24)-(4.25)$, it is suffices to prove that $\left(f_{n}\left(x, T_{n}\left(u_{n}\right)\right)\right)_{n}$ and $\left(\left|T_{n}\left(u_{n}\right)\right|^{s(x)-1} T_{n}\left(u_{n}\right)\right)_{n}$ are uniformly equi-integrable. Let $h \geq R$, by taking $v_{n}=\varphi\left(u_{n}\right)\left|T_{h+1}\left(u_{n}\right)-T_{h}\left(u_{n}\right)\right|$ as a test function in (4.2), and since $v_{n}$ have the same sign as $u_{n}$, we have

$$
\begin{aligned}
& \quad \sum_{i=1}^{N} \int_{\Omega} a_{i}\left(x, T_{n}\left(u_{n}\right), \nabla u_{n}\right)\left(D^{i} T_{h+1}\left(u_{n}\right)-D^{i} T_{h}\left(u_{n}\right)\right) \varphi\left(u_{n}\right) d x \\
& \quad+(\theta-1) \sum_{i=1}^{N} \int_{\Omega} \frac{a_{i}\left(x, T_{n}\left(u_{n}\right), \nabla u_{n}\right) D^{i} u_{n}}{\left(1+\left|u_{n}\right|\right)^{\theta}}\left|T_{h+1}\left(u_{n}\right)-T_{h}\left(u_{n}\right)\right| d x \\
& \quad+\int_{\Omega}\left|T_{n}\left(u_{n}\right)\right|^{s(x)}\left|T_{h+1}\left(u_{n}\right)-T_{h}\left(u_{n}\right)\right|\left|\varphi\left(u_{n}\right)\right| d x \\
& \leq \int_{\Omega}\left|f_{n}\left(x, T_{n}\left(u_{n}\right)\right)\right|\left|T_{h+1}\left(u_{n}\right)-T_{h}\left(u_{n}\right)\right| \varphi\left(u_{n}\right) \mid d x .
\end{aligned}
$$

We have $\left|\varphi\left(u_{n}\right)\right| \geq \frac{1}{2}$ on the set $\left\{h \leq\left|u_{n}\right|\right\}$, and thanks to (3.2) we obtain

$$
\begin{aligned}
& \sum_{i=1}^{N} \int_{\Omega} a_{i}\left(x, T_{n}\left(u_{n}\right), \nabla u_{n}\right)\left(D^{i} T_{h+1}\left(u_{n}\right)-D^{i} T_{h}\left(u_{n}\right)\right)\left|\varphi\left(u_{n}\right)\right| d x \\
& +(\theta-1) \sum_{i=1}^{N} \int_{\Omega} \frac{a_{i}\left(x, T_{n}\left(u_{n}\right), \nabla u_{n}\right) D^{i} u_{n}}{\left(1+\left|u_{n}\right|\right)^{\theta}}\left|T_{h+1}\left(u_{n}\right)-T_{h}\left(u_{n}\right)\right| d x
\end{aligned}
$$




$$
\begin{aligned}
\geq & \frac{1}{4} \sum_{i=1}^{N} \int_{\left\{h<\left|u_{n}\right| \leq h+1\right\}} a_{i}\left(x, T_{n}\left(u_{n}\right), \nabla u_{n}\right) D^{i} u_{n} d x+\frac{\alpha}{4} \sum_{i=1}^{N} \int_{\left\{h<\left|u_{n}\right| \leq h+1\right\}}\left|D^{i} u_{n}\right|^{p_{i}(x)} d x \\
& +\alpha(\theta-1) \sum_{i=1}^{N} \int_{\left\{h+1 \leq\left|u_{n}\right|\right\}} \frac{\left|D^{i} u_{n}\right|^{p_{i}(x)}}{\left(1+\left|u_{n}\right|\right)^{\theta}} d x \\
\geq & \frac{1}{4} \sum_{i=1}^{N} \int_{\left\{h<\left|u_{n}\right| \leq h+1\right\}} a_{i}\left(x, T_{n}\left(u_{n}\right), \nabla u_{n}\right) D^{i} u_{n} d x+C_{5} \sum_{i=1}^{N} \int_{\left\{h+1 \leq\left|u_{n}\right|\right\}} \frac{\left|D^{i} u_{n}\right|^{p_{i}(x)}}{\left(1+\left|u_{n}\right|\right)^{\theta}} d x
\end{aligned}
$$

with $C_{5}=\alpha \cdot \min \left\{\frac{1}{4},(\theta-1)\right\}$. Having in mind (3.5) we conclude that

$$
\begin{aligned}
\text { (4.26) } & \frac{1}{4} \sum_{i=1}^{N} \int_{\left\{h<\left|u_{n}\right| \leq h+1\right\}} a_{i}\left(x, T_{n}\left(u_{n}\right), \nabla u_{n}\right) D^{i} u_{n} d x+C_{5} \sum_{i=1}^{N} \int_{\left\{h+1 \leq\left|u_{n}\right|\right\}} \frac{\left|D^{i} u_{n}\right| p_{i}(x)}{\left(1+\left|u_{n}\right|\right)^{\theta}} d x \\
& +\int_{\Omega}\left|T_{n}\left(u_{n}\right)\right|^{s(x)}\left|T_{h+1}\left(u_{n}\right)-T_{h}\left(u_{n}\right)\right| \varphi\left(u_{n}\right) d x \\
\leq & \int_{\left\{h<\left|u_{n}\right|\right\}}|g(x)|\left|T_{h+1}\left(u_{n}\right)-T_{h}\left(u_{n}\right)\right| d x \\
& +\int_{\left\{h<\left|u_{n}\right|\right\}}\left|T_{n}\left(u_{n}\right)\right|^{q(x)}\left|T_{h+1}\left(u_{n}\right)-T_{h}\left(u_{n}\right)\right|\left|\varphi\left(u_{n}\right)\right| d x .
\end{aligned}
$$

For the second term on the left-hand side of (4.26), thanks to Poincaré's inequality we have

$$
\begin{aligned}
& C_{5} \sum_{i=1}^{N} \int_{\left\{h \leq\left|u_{n}\right|\right\}} \frac{\left|D^{i} u_{n}\right| p_{i}(x)}{\left(1+\left|u_{n}\right|\right)^{\theta}} d x \\
\geq & C_{5} \sum_{i=1}^{N} \int_{\left\{h \leq\left|u_{n}\right|\right\}} \frac{\left|D^{i} u_{n}\right|_{i}^{-}}{\left(1+\left|u_{n}\right|\right)^{\theta}} d x-C_{5} N \operatorname{meas}\left\{h \leq\left|u_{n}\right|\right\} \\
= & C_{5} \sum_{i=1}^{N} \int_{\Omega}\left|D^{i} \int_{\left|T_{h}\left(u_{n}\right)\right|}^{\left|u_{n}\right|} \frac{d s}{(1+s)^{\frac{\theta}{p_{i}^{-}}}}\right|^{p_{i}^{-}} d x-C_{5} N \operatorname{meas}\left\{h \leq\left|u_{n}\right|\right\} \\
\geq & C_{6} \sum_{i=1}^{N} \int_{\Omega}\left|\int_{\left|T_{h}\left(u_{n}\right)\right|}^{\left|u_{n}\right|} \frac{d s}{(1+s)^{\frac{\theta}{p_{i}^{-}}}}\right|^{p_{i}^{-}} d x-C_{5} N \operatorname{meas}\left\{h \leq\left|u_{n}\right|\right\} \\
\geq & C_{6} \sum_{i=1}^{N} \int_{\left\{h \leq\left|u_{n}\right|\right\}} \frac{\left(\left|u_{n}\right|-\left|T_{h}\left(u_{n}\right)\right|\right)^{p_{i}^{-}}}{\left(1+\left|u_{n}\right|\right)^{\theta}} d x-C_{5} N \operatorname{meas}\left\{h \leq\left|u_{n}\right|\right\} \\
\geq & C_{7} \sum_{i=1}^{N} \int_{\left\{h \leq\left|u_{n}\right|\right\}}\left|u_{n}\right|^{p_{i}^{-}-\theta} d x-C_{6} \sum_{i=1}^{N} \int_{\left\{h \leq\left|u_{n}\right|\right\}} \frac{h^{p_{i}^{-}}}{\left(1+\left|u_{n}\right|\right)^{\theta}} d x \\
& -C_{5} N \operatorname{meas}\left\{h \leq\left|u_{n}\right|\right\} .
\end{aligned}
$$


Having in mind (4.26), we conclude that

$$
\begin{aligned}
& \quad \frac{1}{4} \sum_{i=1}^{N} \int_{\left\{h<\left|u_{n}\right| \leq h+1\right\}} a_{i}\left(x, T_{n}\left(u_{n}\right), \nabla u_{n}\right) D^{i} u_{n} d x+C_{7} \sum_{i=1}^{N} \int_{\left\{h \leq\left|u_{n}\right|\right\}}\left|u_{n}\right|^{p_{i}^{-}-\theta} d x \\
& \quad+\int_{\left\{h<\left|u_{n}\right|\right\}}\left|T_{n}\left(u_{n}\right)\right|^{s(x)}\left|T_{h+1}\left(u_{n}\right)-T_{h}\left(u_{n}\right)\right| \varphi\left(u_{n}\right) d x \\
& \leq \int_{\left\{h<\left|u_{n}\right|\right\}}|g(x)| d x+\int_{\left\{h<\left|u_{n}\right|\right\}}\left|T_{n}\left(u_{n}\right)\right|^{q(x)}\left|T_{h+1}\left(u_{n}\right)-T_{h}\left(u_{n}\right)\right|\left|\varphi\left(u_{n}\right)\right| d x \\
& \quad+C_{6} \sum_{i=1}^{N} \int_{\left\{h<\left|u_{n}\right|\right\}} \frac{h^{p_{i}^{-}}}{\left(1+\left|u_{n}\right|\right)^{\theta}} d x+C_{5} N \operatorname{meas}\left\{h \leq\left|u_{n}\right|\right\} .
\end{aligned}
$$

Since $q(x)<\max \left(s(x), \underline{p}^{+}-\theta\right)$, and in view of Young's inequality we have

$$
\begin{aligned}
& \int_{\left\{h<\left|u_{n}\right|\right\}}\left|T_{n}\left(u_{n}\right)\right|^{q(x)}\left|T_{h+1}\left(u_{n}\right)-T_{h}\left(u_{n}\right)\right|\left|\varphi\left(u_{n}\right)\right| d x \\
\leq & \frac{C_{7}}{2} \sum_{i=1}^{N} \int_{\left\{h \leq\left|u_{n}\right|\right\}}\left|u_{n}\right|^{p_{i}^{-}-\theta} d x+C_{8} \int_{\left\{h<\left|u_{n}\right|\right\}}\left|T_{h+1}\left(u_{n}\right)-T_{h}\left(u_{n}\right)\right| d x \\
& +\frac{1}{2} \int_{\left\{h<\left|u_{n}\right|\right\}}\left|T_{n}\left(u_{n}\right)\right|^{s(x)}\left|T_{h+1}\left(u_{n}\right)-T_{h}\left(u_{n}\right)\right|\left|\varphi\left(u_{n}\right)\right| d x,
\end{aligned}
$$

and thanks to (4.23), we have

$$
\begin{aligned}
\varepsilon_{1}(h) & =\sum_{i=1}^{N} \int_{\left\{h<\left|u_{n}\right|\right\}} \frac{h^{p_{i}^{-}}}{\left(1+\left|u_{n}\right|\right)^{\theta}} d x \leq \sum_{i=1}^{N} h^{p_{i}^{-}-\theta} \operatorname{meas}\left\{h<\left|u_{n}\right|\right\} \\
& \leq N h^{\underline{p}^{+}-\theta} \operatorname{meas}\left\{h<\left|u_{n}\right|\right\} \\
& =\frac{N h^{\underline{p}^{+}-1} \operatorname{meas}\left\{h<\left|u_{n}\right|\right\}}{h^{\theta-1}} \\
& \leq \frac{N C_{4}}{h^{\theta-1}} \rightarrow 0 \text { as } h \rightarrow \infty .
\end{aligned}
$$

Also, we have meas $\left\{\left|u_{n}\right|>h\right\}$ goes to zero, as $h$ tends to infinity, and since $g(x) \in$ $L^{1}(\Omega)$ we conclude that

$$
\varepsilon_{2}(h)=\int_{\left\{h<\left|u_{n}\right|\right\}}|g(x)| d x+C_{5} N \text { meas }\left\{h \leq\left|u_{n}\right|\right\} \rightarrow 0 \text { as } h \rightarrow \infty .
$$

It follows that

$$
\begin{aligned}
& \frac{1}{4} \sum_{i=1}^{N} \int_{\left\{h<\left|u_{n}\right| \leq h+1\right\}} a_{i}\left(x, T_{n}\left(u_{n}\right), \nabla u_{n}\right) D^{i} u_{n} d x \\
& +\frac{C_{7}}{2} \sum_{i=1}^{N} \int_{\left\{h \leq\left|u_{n}\right|\right\}}\left|u_{n}\right|^{p_{i}^{-}-\theta} d x+\frac{1}{2} \int_{\left\{h+1<\left|u_{n}\right|\right\}}\left|T_{n}\left(u_{n}\right)\right|^{s(x)} d x \\
\leq & C_{8} \int_{\left\{h<\left|u_{n}\right|\right\}}\left|T_{h+1}\left(u_{n}\right)-T_{h}\left(u_{n}\right)\right| d x+\varepsilon_{3}(h) \\
\leq & \varepsilon_{4}(h) .
\end{aligned}
$$


We conclude that

$$
\lim _{h \rightarrow \infty}\left(\int_{\left\{h+1<\left|u_{n}\right|\right\}}\left|T_{n}\left(u_{n}\right)\right|^{s(x)} d x+\int_{\left\{h+1<\left|u_{n}\right|\right\}}\left|T_{n}\left(u_{n}\right)\right|^{q(x)} d x\right)=0,
$$

therefore, thanks to (4.28) we have for any $\delta>0$, there exists $h(\delta)>1$ such that

$$
\int_{\left\{h(\delta)<\left|u_{n}\right|\right\}}\left|T_{n}\left(u_{n}\right)\right|^{s(x)} d x+\int_{\left\{h(\delta)<\left|u_{n}\right|\right\}}\left|T_{n}\left(u_{n}\right)\right|^{q(x)} d x \leq \frac{\delta}{2} .
$$

On the other hand, for any measurable subset $E \subseteq \Omega$ we have

$$
\begin{aligned}
& \int_{E}\left|T_{n}\left(u_{n}\right)\right|^{s(x)} d x+\int_{E}\left|T_{n}\left(u_{n}\right)\right|^{q(x)} d x \\
\leq & \int_{\left\{h(\delta)<\left|u_{n}\right|\right\}}\left|T_{n}\left(u_{n}\right)\right|^{s(x)} d x+\int_{\left\{h(\delta)<\left|u_{n}\right|\right\}}\left|T_{n}\left(u_{n}\right)\right|^{q(x)} d x \\
& +\int_{E}\left|T_{h(\delta)}\left(u_{n}\right)\right|^{s(x)} d x+\int_{E}\left|T_{h(\delta)}\left(u_{n}\right)\right|^{q(x)} d x .
\end{aligned}
$$

It's clear that, there exists $\beta(\delta)>0$ such that for any $E \subseteq \Omega$ with meas $(E) \leq \beta(\delta)$ we have

$$
\int_{E}\left|T_{h(\delta)}\left(u_{n}\right)\right|^{s(x)} d x+\int_{E}\left|T_{h(\delta)}\left(u_{n}\right)\right|^{q(x)} d x \leq \frac{\delta}{2} .
$$

Finally, by combining (4.29), (4.30) and (4.31), we obtain (4.32)

$$
\int_{E}\left|T_{n}\left(u_{n}\right)\right|^{s(x)} d x+\int_{E}\left|T_{n}\left(u_{n}\right)\right|^{q(x)} d x \leq \delta \text { for any } E \subset \Omega \text { such that meas }(E) \leq \beta(\delta) \text {. }
$$

Consequently, $\left(\left|T_{n}\left(u_{n}\right)\right|^{s(x)-1} T_{n}\left(u_{n}\right)\right)_{n}$ and $\left(\left|T_{n}\left(u_{n}\right)\right|^{q(x)-1} T_{n}\left(u_{n}\right)\right)_{n}$ are uniformly equiintegrable, and in view of the growth condition (3.5) we have

$$
\left|f_{n}\left(x, T_{n}\left(u_{n}\right)\right)\right| \leq g(x)+\left|T_{n}\left(u_{n}\right)\right|^{q(x)},
$$

with $g(x) \in L^{1}(\Omega)$, then $\left(f_{n}\left(x, T_{n}\left(u_{n}\right)\right)\right)_{n}$ is also uniformly equi-integrable. According to Vitali's theorem, the statements (4.24) and (4.25) are concluded. Moreover, in view of (4.27) we have

$$
\lim _{h \rightarrow \infty} \limsup _{n \rightarrow \infty} \sum_{i=1}^{N} \int_{\left\{h<\left|u_{n}\right| \leq h+1\right\}} a_{i}\left(x, T_{n}\left(u_{n}\right), \nabla u_{n}\right) D^{i} u_{n} d x=0 .
$$

Step 4: strong convergence of truncations. Let $h>k \geq 1$, and we set $\psi_{h}\left(u_{n}\right)=$ $\left(1-\left|T_{1}\left(u_{n}-T_{h}\left(u_{n}\right)\right)\right|\right)$. By taking $\left(T_{k}\left(u_{n}\right)-T_{k}(u)\right) \psi_{h}\left(u_{n}\right) \in W_{0}^{1, \vec{p}(\cdot)}(\Omega)$ as a test function in (4.2) we obtain

$$
\begin{aligned}
& \sum_{i=1}^{N} \int_{\Omega} a_{i}\left(x, T_{n}\left(u_{n}\right), \nabla u_{n}\right)\left(D^{i} T_{k}\left(u_{n}\right)-D^{i} T_{k}(u)\right) \psi_{h}\left(u_{n}\right) d x \\
& -\sum_{i=1}^{N} \int_{\left\{h \leq\left|u_{n}\right| \leq h+1\right\}} a_{i}\left(x, T_{n}\left(u_{n}\right), \nabla u_{n}\right) D^{i} u_{n}\left|T_{k}\left(u_{n}\right)-T_{k}(u)\right| d x \\
& +\int_{\Omega}\left|T_{n}\left(u_{n}\right)\right|^{s(x)-1} T_{n}\left(u_{n}\right)\left(T_{k}\left(u_{n}\right)-T_{k}(u)\right) \psi_{h}\left(u_{n}\right) d x
\end{aligned}
$$




$$
=\int_{\Omega} f_{n}\left(x, T_{n}\left(u_{n}\right)\right)\left(T_{k}\left(u_{n}\right)-T_{k}(u)\right) \psi_{h}\left(u_{n}\right) d x
$$

It follows that

$$
\begin{aligned}
& \sum_{i=1}^{N} \int_{\Omega} a_{i}\left(x, T_{n}\left(u_{n}\right), \nabla u_{n}\right)\left(D^{i} T_{k}\left(u_{n}\right)-D^{i} T_{k}(u)\right) \psi_{h}\left(u_{n}\right) d x \\
\leq & \int_{\Omega}\left|f_{n}\left(x, T_{n}\left(u_{n}\right)\right)\right|\left|T_{k}\left(u_{n}\right)-T_{k}(u)\right| d x+\int_{\Omega}\left|T_{n}\left(u_{n}\right)\right|^{s(x)}\left|T_{k}\left(u_{n}\right)-T_{k}(u)\right| d x \\
& +\sum_{i=1}^{N} \int_{\left\{h \leq\left|u_{n}\right| \leq h+1\right\}} a_{i}\left(x, T_{n}\left(u_{n}\right), \nabla u_{n}\right) D^{i} u_{n}\left|T_{k}\left(u_{n}\right)-T_{k}(u)\right| d x .
\end{aligned}
$$

For the first and second terms on the right-hand side of (4.34), we have $T_{k}\left(u_{n}\right) \rightarrow T_{k}(u)$

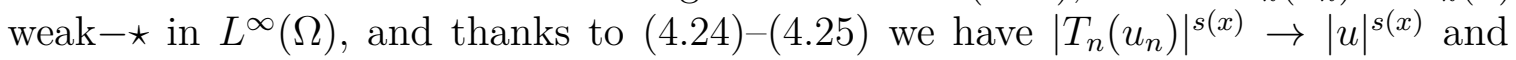
$f_{n}\left(x, T_{n}\left(u_{n}\right)\right) \rightarrow f(x, u)$ strongly in $L^{1}(\Omega)$, then

$$
\varepsilon_{5}(n)=\int_{\Omega}\left|T_{n}\left(u_{n}\right)\right|^{s(x)}\left|T_{k}\left(u_{n}\right)-T_{k}(u)\right| d x \rightarrow 0 \text { as } n \rightarrow \infty
$$

and

$$
\varepsilon_{6}(n)=\int_{\Omega}\left|f_{n}\left(x, T_{n}\left(u_{n}\right)\right)\right|\left|T_{k}\left(u_{n}\right)-T_{k}(u)\right| d x \rightarrow 0 \text { as } n \rightarrow \infty .
$$

On the other hand, according to (4.33) we have

$$
\begin{aligned}
\varepsilon_{7}(h) & =\sum_{i=1}^{N} \int_{\left\{h<\left|u_{n}\right| \leq h+1\right\}} a_{i}\left(x, T_{n}\left(u_{n}\right), \nabla u_{n}\right) D^{i} u_{n}\left|T_{k}\left(u_{n}\right)-T_{k}(u)\right| d x \\
& \leq 2 k \sum_{i=1}^{N} \int_{\left\{h<\left|u_{n}\right| \leq h+1\right\}} a_{i}\left(x, T_{n}\left(u_{n}\right), \nabla u_{n}\right) D^{i} u_{n} d x \rightarrow 0 \text { as } h \rightarrow \infty .
\end{aligned}
$$

By combining (4.34) and (4.35)-(4.37) we conclude that

$$
\sum_{i=1}^{N} \int_{\Omega} a_{i}\left(x, T_{n}\left(u_{n}\right), \nabla u_{n}\right)\left(D^{i} T_{k}\left(u_{n}\right)-D^{i} T_{k}(u)\right) \psi_{h}\left(u_{n}\right) d x \leq \varepsilon_{7}(n, h) .
$$

For the term on the left-hand side of (4.38), since $a_{i}(x, s, 0)=0$, it follows that

$$
\begin{aligned}
& \sum_{i=1}^{N} \int_{\Omega} a_{i}\left(x, T_{n}\left(u_{n}\right), \nabla u_{n}\right)\left(D^{i} T_{k}\left(u_{n}\right)-D^{i} T_{k}(u)\right) \psi_{h}\left(u_{n}\right) d x \\
= & \sum_{i=1}^{N} \int_{\left\{\left|u_{n}\right| \leq k\right\}} a_{i}\left(x, T_{k}\left(u_{n}\right), \nabla T_{k}\left(u_{n}\right)\right)\left(D^{i} T_{k}\left(u_{n}\right)-D^{i} T_{k}(u)\right) d x \\
& -\sum_{i=1}^{N} \int_{\left\{k<\left|u_{n}\right| \leq h+1\right\}} a_{i}\left(x, T_{h+1}\left(u_{n}\right), \nabla T_{h+1}\left(u_{n}\right)\right) D^{i} T_{k}(u) \psi_{h}\left(u_{n}\right) d x \\
= & \sum_{i=1}^{N} \int_{\Omega}\left(a_{i}\left(x, T_{k}\left(u_{n}\right), \nabla T_{k}\left(u_{n}\right)\right)-a_{i}\left(x, T_{k}\left(u_{n}\right), \nabla T_{k}(u)\right)\right)\left(D^{i} T_{k}\left(u_{n}\right)-D^{i} T_{k}(u)\right) d x
\end{aligned}
$$




$$
\begin{aligned}
& +\sum_{i=1}^{N} \int_{\Omega} a_{i}\left(x, T_{k}\left(u_{n}\right), \nabla T_{k}(u)\right)\left(D^{i} T_{k}\left(u_{n}\right)-D^{i} T_{k}(u)\right) d x \\
& -\sum_{i=1}^{N} \int_{\left\{k<\left|u_{n}\right| \leq h+1\right\}} a_{i}\left(x, T_{h+1}\left(u_{n}\right), \nabla T_{h+1}\left(u_{n}\right)\right) D^{i} T_{k}(u) \psi_{h}\left(u_{n}\right) d x .
\end{aligned}
$$

For the second term on the right-hand side of (4.39), we have $T_{k}\left(u_{n}\right) \rightarrow T_{k}(u)$ in $L^{p_{i}(\cdot)}(\Omega)$, then, $a_{i}\left(x, T_{k}\left(u_{n}\right), \nabla T_{k}(u)\right) \rightarrow a_{i}\left(x, T_{k}(u), \nabla T_{k}(u)\right)$ strongly in $L^{p_{i}^{\prime}(\cdot)}(\Omega)$, and since $D^{i} T_{k}\left(u_{n}\right)$ converges to $D^{i} T_{k}(u)$ weakly in $L^{p^{2}(\cdot)}(\Omega)$, we obtain

$$
\varepsilon_{8}(n)=\sum_{i=1}^{N} \int_{\Omega} a_{i}\left(x, T_{k}\left(u_{n}\right), \nabla T_{k}(u)\right)\left(D^{i} T_{k}\left(u_{n}\right)-D^{i} T_{k}(u)\right) d x \rightarrow 0 \text { as } n \rightarrow \infty .
$$

Concerning the third term on the right-hand side of (4.39), we have $\left(\mid a_{i}\left(x, T_{h+1}\left(u_{n}\right)\right.\right.$, $\left.\left.\nabla T_{h+1}\left(u_{n}\right)\right) \mid\right)_{n}$ is bounded in $L^{p_{i}^{\prime}(\cdot)}(\Omega)$, then there exists $\nu_{i} \in L^{p_{i}^{\prime}(\cdot)}(\Omega)$ such that $\left|a_{i}\left(x, T_{h+1}\left(u_{n}\right), \nabla T_{h+1}\left(u_{n}\right)\right)\right| \rightarrow \nu_{i}$ weakly in $L^{p_{i}^{\prime}(\cdot)}(\Omega)$ for any $i=1, \ldots, N$. Therefore,

$$
\begin{aligned}
\varepsilon_{9}(n) & \leq\left|\sum_{i=1}^{N} \int_{\left\{k<\left|u_{n}\right| \leq h+1\right\}} a_{i}\left(x, T_{h+1}\left(u_{n}\right), \nabla T_{h+1}\left(u_{n}\right)\right) D^{i} T_{k}(u) \psi_{h}\left(u_{n}\right) d x\right| \\
& \leq \sum_{i=1}^{N} \int_{\left\{k<\left|u_{n}\right| \leq h+1\right\}}\left|a_{i}\left(x, T_{h+1}\left(u_{n}\right), \nabla T_{h+1}\left(u_{n}\right)\right)\right|\left|D^{i} T_{k}(u)\right| d x \\
& \rightarrow \sum_{i=1}^{N} \int_{\{k<|u| \leq h+1\}} \nu_{i}\left|D^{i} T_{k}(u)\right| d x=0 \text { as } n \rightarrow \infty .
\end{aligned}
$$

By combining (4.38)-(4.41), we conclude that

$$
\begin{aligned}
& \sum_{i=1}^{N} \int_{\Omega}\left(a_{i}\left(x, T_{k}\left(u_{n}\right), \nabla T_{k}\left(u_{n}\right)\right)-a_{i}\left(x, T_{k}\left(u_{n}\right), \nabla T_{k}(u)\right)\right)\left(D^{i} T_{k}\left(u_{n}\right)-D^{i} T_{k}(u)\right) d x \\
\leq & \varepsilon_{10}(n, h) .
\end{aligned}
$$

In view of Lebesgue dominated convergence theorem, we have $T_{k}\left(u_{n}\right) \rightarrow T_{k}(u)$ strongly in $L^{\underline{p}}(\Omega)$. Thus, by letting $n$ then $h$ tend to infinity we deduce that

$$
\begin{aligned}
& \sum_{i=1}^{N} \int_{\Omega}\left(a_{i}\left(x, T_{k}\left(u_{n}\right), \nabla T_{k}\left(u_{n}\right)\right)-a_{i}\left(x, T_{k}\left(u_{n}\right), \nabla T_{k}(u)\right)\right)\left(D^{i} T_{k}\left(u_{n}\right)-D^{i} T_{k}(u)\right) d x \\
& +\int_{\Omega}\left(\left|T_{k}\left(u_{n}\right)\right|^{\underline{p}^{+}-2} T_{k}\left(u_{n}\right)-\left|T_{k}(u)\right|^{\underline{p}^{+}-2} T_{k}(u)\right)\left(T_{k}\left(u_{n}\right)-T_{k}(u)\right) d x \rightarrow 0 \text { as } n \rightarrow \infty .
\end{aligned}
$$

In view of Lemma 4.2, we conclude that

$$
\left\{\begin{array}{l}
T_{k}\left(u_{n}\right) \rightarrow T_{k}(u) \text { strongly in } W_{0}^{1, \vec{p}(\cdot)}(\Omega) \\
D^{i} u_{n} \rightarrow D^{i} u \text { a.e. in } \Omega \text { for } i=1, \ldots, N
\end{array}\right.
$$


Moreover, we have $a_{i}\left(x, T_{n}\left(u_{n}\right), \nabla u_{n}\right) D^{i} u_{n}$ tends to $a_{i}(x, u, \nabla u) D^{i} u$ almost everywhere in $\Omega$, and in view of Fatou's lemma and (4.33), we conclude that

$$
\begin{aligned}
& \lim _{h \rightarrow \infty} \sum_{i=1}^{N} \int_{\{h<|u|<h+1\}} a_{i}(x, u, \nabla u) D^{i} u d x \\
\leq & \lim _{h \rightarrow \infty} \liminf _{n \rightarrow \infty} \sum_{i=1}^{N} \int_{\left\{h<\left|u_{n}\right|<h+1\right\}} a_{i}\left(x, T_{n}\left(u_{n}\right), \nabla u_{n}\right) D^{i} u_{n} d x \\
\leq & \lim _{h \rightarrow \infty} \limsup _{n \rightarrow \infty} \sum_{i=1}^{N} \int_{\left\{h<\left|u_{n}\right|<h+1\right\}} a_{i}\left(x, T_{n}\left(u_{n}\right), \nabla u_{n}\right) D^{i} u_{n} d x=0,
\end{aligned}
$$

which prove (4.1).

Step 5: passage to the limit. Let $\varphi \in W_{0}^{1, \vec{p}(\cdot)}(\Omega) \cap L^{\infty}(\Omega)$, and choosing $S(\cdot)$ be a smooth function in $C_{0}^{1}(\mathbb{R})$ such that $\operatorname{supp}(S(\cdot)) \subseteq[-M, M]$ for some $M \geq 0$.

By taking $S\left(u_{n}\right) \varphi \in W_{0}^{1, \vec{p}(\cdot)}(\Omega) \cap L^{\infty}(\Omega)$ as a test function in the approximate problem (4.2), we obtain

$$
\begin{aligned}
& \sum_{i=1}^{N} \int_{\Omega} a_{i}\left(x, T_{n}\left(u_{n}\right), \nabla u_{n}\right)\left(D^{i} u_{n} S^{\prime}\left(u_{n}\right) \varphi+S\left(u_{n}\right) D^{i} \varphi\right) d x \\
& +\int_{\Omega}\left|T_{n}\left(u_{n}\right)\right|^{s(x)-1} T_{n}\left(u_{n}\right) S\left(u_{n}\right) \varphi d x=\int_{\Omega} f_{n}\left(x, T_{n}\left(u_{n}\right)\right) S\left(u_{n}\right) \varphi d x .
\end{aligned}
$$

For the first term on the left-hand side of (4.43), we have

$$
\begin{aligned}
& \sum_{i=1}^{N} \int_{\Omega} a_{i}\left(x, T_{n}\left(u_{n}\right), \nabla u_{n}\right)\left(D^{i} u_{n} S^{\prime}\left(u_{n}\right) \varphi+S\left(u_{n}\right) D^{i} \varphi\right) d x \\
= & \sum_{i=1}^{N} \int_{\Omega} a_{i}\left(x, T_{M}\left(u_{n}\right), \nabla T_{M}\left(u_{n}\right)\right)\left(S^{\prime}\left(u_{n}\right) \varphi D^{i} T_{M}\left(u_{n}\right)+S\left(T_{M}\left(u_{n}\right)\right) D^{i} \varphi\right) d x,
\end{aligned}
$$

in view of (4.42), we have $\left(a_{i}\left(x, T_{M}\left(u_{n}\right), \nabla T_{M}\left(u_{n}\right)\right)\right)_{n}$ is bounded in $L^{p^{\prime}(\cdot)}(\Omega)$, and since $a_{i}\left(x, T_{M}\left(u_{n}\right), \nabla T_{M}\left(u_{n}\right)\right)$ tends to $a_{i}\left(x, T_{M}(u), \nabla T_{M}(u)\right)$ almost everywhere in $\Omega$, it follows that

$$
a_{i}\left(x, T_{M}\left(u_{n}\right), \nabla T_{M}\left(u_{n}\right)\right) \rightarrow a_{i}\left(x, T_{M}(u), \nabla T_{M}(u)\right) \text { in } L^{p_{i}^{\prime}(\cdot)}(\Omega),
$$

and since $\left(S^{\prime}\left(u_{n}\right) \varphi D^{i} T_{M}\left(u_{n}\right)+S\left(T_{M}\left(u_{n}\right)\right) D^{i} \varphi\right) \rightarrow\left(S^{\prime}(u) \varphi D^{i} T_{M}(u)+S\left(T_{M}(u)\right) D^{i} \varphi\right)$ strongly in $L^{p_{i}(\cdot)}(\Omega)$, we deduce that

$$
\begin{aligned}
& \lim _{n \rightarrow \infty} \sum_{i=1}^{N} \int_{\Omega} a_{i}\left(x, T_{n}\left(u_{n}\right), \nabla u_{n}\right)\left(D^{i} u_{n} S^{\prime}\left(u_{n}\right) \varphi+S\left(u_{n}\right) D^{i} \varphi\right) d x \\
= & \lim _{n \rightarrow \infty} \sum_{i=1}^{N} \int_{\Omega} a_{i}\left(x, T_{M}\left(u_{n}\right), \nabla T_{M}\left(u_{n}\right)\right)\left(D^{i} T_{M}\left(u_{n}\right) S^{\prime}\left(u_{n}\right) \varphi+S\left(T_{M}\left(u_{n}\right)\right) D^{i} \varphi\right) d x \\
= & \sum_{i=1}^{N} \int_{\Omega} a_{i}\left(x, T_{M}(u), \nabla T_{M}(u)\right)\left(D^{i} T_{M}(u) S^{\prime}(u) \varphi+S\left(T_{M}(u)\right) D^{i} \varphi\right) d x
\end{aligned}
$$




$$
=\sum_{i=1}^{N} \int_{\Omega} a_{i}(x, u, \nabla u)\left(D^{i} u S^{\prime}(u) \varphi+S(u) D^{i} \varphi\right) d x .
$$

Concerning the second term on the right-hand side of (4.43), we have $S\left(T_{M}\left(u_{n}\right)\right) \varphi \rightarrow$ $S\left(T_{M}(u)\right) \varphi$ weak-* in $L^{\infty}(\Omega)$, and thanks to $(4.24)$, we have $\left|T_{n}\left(u_{n}\right)\right|^{s(x)-1} T_{n}\left(u_{n}\right) \rightarrow$ $|u|^{s(x)-1} u$ strongly in $L^{1}(\Omega)$, it follows that

$$
\begin{aligned}
& \lim _{n \rightarrow \infty} \int_{\Omega}\left|T_{n}\left(u_{n}\right)\right|^{s(x)-1} T_{n}\left(u_{n}\right) S\left(T_{M}\left(u_{n}\right)\right) \varphi d x=\int_{\Omega}|u|^{s(x)-1} u S\left(T_{M}(u)\right) \varphi d x \\
= & \int_{\Omega}|u|^{s(x)-1} u S(u) \varphi d x .
\end{aligned}
$$

Similarly, thanks to $(4.25)$ we have $f_{n}\left(x, T_{n}\left(u_{n}\right)\right) \rightarrow f(x, u)$ strongly in $L^{1}(\Omega)$ then (4.46)

$$
\lim _{n \rightarrow \infty} \int_{\Omega} f_{n}\left(x, T_{n}\left(u_{n}\right)\right) S\left(T_{M}\left(u_{n}\right)\right) \varphi d x=\int_{\Omega} f(x, u) S\left(T_{M}(u)\right) \varphi d x=\int_{\Omega} f(x, u) S(u) \varphi d x .
$$

By combining (4.43) and (4.44)-(4.46), we conclude that

$$
\begin{aligned}
& \sum_{i=1}^{N} \int_{\Omega} a_{i}(x, u, \nabla u)\left(D^{i} u S^{\prime}(u) \varphi+S(u) D^{i} \varphi\right) d x+\int_{\Omega}|u|^{s(x)-1} u S(u) \varphi d x \\
= & \int_{\Omega} f(x, u) S(u) \varphi d x .
\end{aligned}
$$

which complete the proof of the Theorem 4.1.

\section{REFERENCES}

[1] S. Antontsev and M. Chipot, Anisotropic equations: uniqueness and existence results, Differential Integral Equations 21 (2008), 401-419.

[2] M. Ben-Cheikh-Ali and O. Guibé, Nonlinear and non-coercive elliptic problems with integrable data, Adv. Math. Sci. Appl. 16 (2006), 275-297.

[3] M. B. Benboubker, E. Azroul and A. Barbara, Quasilinear elliptic problems with nonstandard growths, Electron. J. Differential Equations (2011), 1-16.

[4] M. B. Benboubker, H. Hjiaj and S. Ouaro, Entropy solutions to nonlinear elliptic anisotropic problem with variable exponent, J. Appl. Anal. Comput. 4 (2014), 245-270.

[5] M. Bendahmane, M. Chrif and S. E. Manouni, An approximation result in generalized anisotripic sobolev spaces and application, J. Anal. Appl. 30 (2011), 341-353.

[6] M. Bendahmane and P. Wittbold, Renormalized solutions for nonlinear elliptic equations with variable exponents and $l 1$ data, Nonlinear Anal. 70 (2009), 567-583.

[7] L. Boccardo, D. Giachetti, J. I. Dias and F. Murat, Existence and regularity of renormalized solutions for some elliptic problems involving derivations of nonlinear terms, J. Differential Equations 106 (1993), 215-237.

[8] R. Di-Nardo and F. Feo, Existence and uniqueness for nonlinear anisotropic elliptic equations, Arch. Math. (Basel) 102 (2014), 141-153.

[9] R. Di-Nardo, F. Feo and O. Guibé, Uniqueness result for nonlinear anisotropic elliptic equations, Adv. Differential Equations 18 (2013), 433-458.

[10] L. Diening, P. Harjulehto, P. Hästö and M. Råžička, Lebesgue and Sobolev Spaces with Variable Exponents, Lecture Notes in Mathematics 2017, Springer, Heidelberg, Germany, 2011.

[11] X. L. Fan and Q. H. Zhang, Existence for $p(x)$-Laplacien Dirichlet problem, Nonlinear Anal. 52 (2003), 1843-1852. 
[12] J. L. Lions, Quelques Methodes de Résolution des Problèmes aux Limites non Linéaires, Dunod et Gauthiers-Villars, Paris, 1969.

[13] M. Mihailescu, P. Pucci and V. Radulescu, Eigenvalue problems for anisotropic quasilinear elliptic equations with variable exponent, J. Math. Anal. Appl. 340 (2008), 687-698.

[14] K. Rajagopal and M. Råžička, Mathematical modelling of electro-rheological fluids, Contin. Mech. Thermodyn. 13 (2001), 59-78.

[15] M. Råžička, Electrorheological Fluids: Modeling and Mathematical Theory, Lecture Notes in Mathematics 1748, Springer, Berlin, 2000.

[16] J. F. Rodrigues, Obstacles Problems in Mathematical Physics, North-Holland, Amsterdam, 1991.

[17] L. Zhao, P. Zhao and X. Xie, Existence and multiplicty of solutions for vivergence type elliptic equations, Electron. J. Differential Equations 43 (2009), 1-9.

${ }^{1}$ LAMA LABORATORY, Department of MAThematics,

University of Sidi Mohamed Ben Abdellah, Faculty of sciences Dhar El Mahraz,

B. P. 1796 Atlas Fez, Morocco

Email address: taghi-med@hotmail.fr

Email address: ahmedmath2001@gmail.com

${ }^{2}$ Department of Mathematics,

University Abdelmalek Essaadi, Faculty of Sciences Tetouan,

B. P. 2121, Tetouan, Morocco

Email address: hjiajhassane@yahoo.fr

${ }^{1}$ LAMA LABORATORY, Department of MATHEMATiCs,

University of Sidi Mohamed Ben Abdellah, Faculty of sciences Dhar El Mahraz, B. P. 1796 Atlas Fez, Morocco

Email address: atouzani07@gmail.com 